\title{
Double cortical stimulation in amyotrophic lateral sclerosis
}

Takanori Yokota, Akiko Yoshino, Akira Inaba, Yukinobu Saito

\begin{abstract}
Objective-Transcranial double magnetic stimulation on the motor cortex was used to investigate central motor tract function in 16 patients with amyotrophic lateral sclerosis, five with spinal muscular atrophy, and 16 age matched normal controls. Methods-Surface EMG responses were recorded from the relaxed abductor pollicis brevis (APB) muscle.

Results-Responses to test stimuli were markedly attenuated by a subthreshold conditioning stimulus given at a condition-test (C-T) interval of 1-4 ms in normal controls and patients with spinal muscular atrophy, but attenuation was mild in patients with amyotrophic lateral sclerosis. In the normal controls this suppression was caused by activation of the intracortical inhibitory mechanism because responses to electrical test stimuli and the $H$ wave were not suppressed by the same magnetic subthreshold conditioning stimulus. In amyotrophic lateral sclerosis the effect of the conditioning cortical stimulus on the $H$ wave was also in the normal range.
\end{abstract}

Conclusion-The intracortical inhibitory mechanism may be impaired in patients with amyotrophic lateral sclerosis.

$(\mathcal{F}$ Neurol Neurosurg Psychiatry 1996;61:596-600)

Keywords: motor evoked potential; magnetic stimulation; amyotrophic lateral sclerosis; double cortical stimulation

The pathogenesis of amyotrophic lateral sclerosis has been an enigma for over a century. A recent hypothesis suggests that the corticomotor neuronal pathways are abnormally excitable in the early stage of amyotrophic lateral sclerosis. ${ }^{12}$ Eisen $^{3}$ showed that the cortical threshold for producing motor evoked potentials (MEPs) early in amyotrophic lateral sclerosis is lower than that in normal subjects. Kaji and Kimura ${ }^{2}$ suggested that at least some fasciculations in amyotrophic lateral sclerosis are driven cortically without hyperexcitability of the motor neurons. We used a double magnetic cortical stimulation technique ${ }^{4}{ }^{5}$ to examine the motor cortical excitability of patients with early amyotrophic lateral sclerosis and compared the results with those for patients with spinal muscular atrophy and age matched normal controls.
Subjects

Sixteen patients with amyotrophic lateral sclerosis (eight men and eight women, mean (SD) age $63.4(6.5)$ years, duration of illness 13.5 (9.7) months), five with spinal muscular atrophy (five men; age 55.5 (5.9), duration of illness $12 \cdot 0(8 \cdot 7)$ years), and 16 age matched normal controls were studied. Selection of patients with amyotrophic lateral sclerosis was based on the following criteria: (1) progressive muscular weakness and atrophy; (2) fasciculations of the affected muscles; (3) clinical signs of pyramidal tract lesion; (4) abnormal needle EMG results characteristic of anterior horn cell disease; (5) absence of sensory signs; sphincter abnormalities, and dementia; (6) absence of conduction block and abnormal temporal dispersion; (7) motor evoked potential (MEP) at rest $>1 \mathrm{mV}$ without temporal dispersion and normal threshold stimulation intensity at rest that produces MEP; (8) MEP latency on electric cortical stimulation of low intensity shorter by more than $1.0 \mathrm{~ms}$ than that in magnetic stimulation for the selection of patients in which magnetic stimuli activate I waves. Selection of patients with spinal muscular atrophy was based on slowly progressive muscular weakness and atrophy without clinical signs of pyramidal tract lesion, as well as on criteria (4)-(8) for amyotrophic lateral sclerosis.

\section{Methods}

After informed consent, the patients and normal controls underwent transcranial magnetic stimulation (TMS). Each subject was seated in a comfortable reclining chair. Surface EMG recordings were made from the APB or flexor carpi radialis (FCR) muscle via surface electrodes attached in a belly tendon montage. Responses were amplified and filtered through Neuropack 4 (Nihon-Koden Ltd, Tokyo), the band pass ranging from 20 to $3 \mathrm{kHz}$. Transcranial stimulation was produced by a Magstim 200 magnetic stimulator (The Magstim Company, Whitland, Dyfed) or a Digitimer D180 high voltage electrical stimulator. For double cortical stimulation study magnetic stimulation was given by a figure of eight shaped coil, each loop having an outer diameter of $9 \mathrm{~cm}$ and a peak magnetic field of $2 \cdot 2 \mathrm{~T}$. The coil was held so that the electrical current in the junction region flowed from the anterior to the posterior direction over the lateral part of the motor strip. For routine MEP study, a round coil (14 $\mathrm{cm}$ outer diameter, 1.5 
T) was used by conventional methods. ${ }^{5}$ The threshold stimulation intensity at rest is defined as the stimulus required to produce a MEP of at least $0.1 \mathrm{mV}$ amplitude in at least $50 \%$ of the trials. The central motor tract conduction time (CMCT) and MEP amplitude: $M$ wave amplitude (MEP:M ratio) were estimated by the conventional method of Eisen and Shybel. ${ }^{5}$

The difference in the MEP latencies produced by electrical and magnetic stimuli was examined while the subjects maintained constant slight voluntary contraction $(2 \%-5 \%$ of maximum) of the APB muscle. Electrical stimulation was given by a Digitimer D180 high voltage electrical stimulator through two $\mathrm{Ag}$ $\mathrm{AgCl}$ EEG electrodes $9 \mathrm{~mm}$ in diameter, fixed at the vertex (cathode) and $7 \mathrm{~cm}$ lateral to the vertex (anode). The intensities of the electrical and magnetic stimulators were adjusted to evoke EMG responses of $1 \mathrm{mV}$.

\section{DOUBLE MAGNETIC STIMULATION}

For double stimulation two magnetic stimulators were connected to the same stimulating coil through a Bistim module (Magstim Company, Whitland, Dyfed, UK). During the examination the subject was asked to keep his or her eyes open so as not to fall asleep. Complete relaxation was ensured by monitoring the real time surface EMG activity at high gain. The subject received auditory feedback through a loudspeaker. The conditioning stimulus was applied to the same point on the scalp as the test stimulus at various conditioning-test (C-T) intervals. The respective intensities of the conditioning and test stimuli were adjusted to $90 \%$ and $130 \%$ of the relaxed threshold which was determined with the same figure of eight coil. In six of the normal subjects, the intensity of conditioning shock was varied from $60 \%$ to $100 \%$ of the threshold. We also compared the effect of a magnetic conditioning stimulus on the test responses evoked by magnetic or electrical test stimuli in four subjects. The intensity of the cortical electrical stimulus was adjusted to a level that produced a response of similar size to that evoked by the magnetic test stimulus at the relaxed APB muscle.

We used a condition-test design to investigate the time course of the effects. Conditioned and non-conditioned test shocks at different $\mathrm{C}-\mathrm{T}$ intervals were intermixed randomly at intervals of four to six seconds. Eight to 12 responses per condition were collected, and their peak to peak amplitudes measured. At each C-T interval, the peak to peak size of

Results of routine $M E P$ study

\begin{tabular}{lccc}
\hline & $\begin{array}{c}\text { ALS } \\
(n=17)\end{array}$ & $\begin{array}{l}S M A \\
(n=5)\end{array}$ & $\begin{array}{l}\text { Normal } \\
\text { subjects } \\
(n=17)\end{array}$ \\
\hline MEP threshold (\%) & $55 \cdot 7(11 \cdot 4)$ & $58 \cdot 8(4 \cdot 5)$ & $53 \cdot 7(7 \cdot 9)$ \\
CMCT (ms) & $6 \cdot 0(1 \cdot 3)$ & $7 \cdot 0(1 \cdot 1)$ & $6 \cdot 3(0 \cdot 8)$ \\
MEP size (mV) & $4 \cdot 2(2 \cdot 1)$ & $4 \cdot 7(1 \cdot 70)$ & $6 \cdot 2(2 \cdot 3)$ \\
M size (mV) & $7 \cdot 2(4 \cdot 4)$ & $7 \cdot 1(3 \cdot 9)$ & $14 \cdot 1(4 \cdot 6)$ \\
MEP:M ratio & $65 \cdot 8(29 \cdot 2)$ & $51 \cdot 8(8 \cdot 3)$ & $45 \cdot 8(15 \cdot 5)$ \\
\hline
\end{tabular}

Values are means (SD). ALS = Amyotrophic lateral sclerosis; SMA = spinal muscular atrophy. the conditioned responses was expressed as a ratio of the size of the unconditioned response (MEP size ratio).

We also examined the correlation between the MEP size ratios at C-T intervals of 2 and $3 \mathrm{~ms}$ and clinical findings, such as duration of illness, grip strength, and $M$ wave size as an indicator for muscle atrophy.

\section{EFFECT OF SUBTHRESHOLD MOTOR CORTICAL} STIMULATION ON THE H REFLEX

We studied change in $\alpha$-motor neuronal excitability after subthreshold magnetic cortical shock by the method of Cowan et al $^{6}$ in six of the normal controls and one patient with amyotrophic lateral sclerosis. The $\mathrm{H}$ reflexes were elicited in the FCR muscle by stimulating the median nerve in the cubital fossa electrically with $1 \mathrm{~ms}$ square wave constant current pulses of about motor threshold intensity. The intensity of the test was adjusted to obtain an $\mathrm{H}$ reflex $30 \%-50 \%$ of the maximal size. Twelve $\mathrm{H}$ reflexes conditioned by a TMS were intermixed randomly with six control $\mathrm{H}$ reflexes for each C-T interval. Magnetic stimulus as the conditioning shock was timed to precede the test shock by corresponding intervals of $0-10 \mathrm{~ms}$ in the magnetic double stimulation of the motor cortex. The time between each subsequent $\mathrm{H}$ reflex was five seconds.

Multiple analysis of variance (MANOVA) was used for the statistical analysis of the differences in MEP size ratios at each C-T interval in normal subjects and patients; and the unpaired $t$ test was used for the analysis of MEP threshold, CMCT, MEP size, $M$ size, and MEP:M ratio between normal subjects and patients.

\section{Results}

The table shows the results of the routine MEP studies. Because of the criteria used for case selection, patients with amyotrophic lateral sclerosis with an early stage of motor tract involvement were selected; CMCT, MEP threshold, and MEP:M ratio did not differ from those of controls although the $M$ wave and MEP sizes were small. Also, in spinal muscular atrophy the MEP and $M$ sizes were small, but CMCT, MEP threshold, and MEP:M ratio were normal.

\section{DOUBLE MAGNETIC STIMULATION}

In normal subjects the size of response to test shock was significantly reduced by conditioning shock at a short C-T interval when the intensity of conditioning shock was $90 \%$ of the threshold in both the APB (fig $1 \mathrm{~A}$ and $\mathrm{C}$ ) and FCR muscles. This suppression was significant $(P<0.01)$ at $C-T$ intervals of $1-4 \mathrm{~ms}$ in the APB muscle and at intervals of $1-3 \mathrm{~ms}$ in the FCR muscle, and was nearly significant at $5 \mathrm{~ms}$ in the APB muscle and at 4 and $5 \mathrm{~ms}$ in the FCR muscle $(P<0.05)$. The suppression was followed by facilitation at a C-T interval of $10 \mathrm{~ms}$ in both muscles $(P<0.01)$. When the intensity of the conditioning shock ranged from $60,70,80,90$, or $100 \%$ of the threshold for excitation, the sizes of responses to condi- 
Figure 1 Effect of subthreshold conditioning stimulation on MEP size in normal subjects and in patients with amyotrophic lateral sclerosis (ALS). (A) MEP wave forms of the non-conditioned test and conditioned responses in normal controls and (B) in patients with amyotrophic lateral sclerosis. (C) Time courses of the effects as ratios of the mean conditioned to nonconditioned MEP size in the normal controls, patients with amyotrophic lateral sclerosis, and patients with spinal muscular atrophy (SMA). Values below 1 represent suppression. Vertical bars are $S D$. Statistical analysis was performed on $M E P$ ratios between patients with amyotrophic lateral sclerosis and normal subjects, and between patients with spinal muscular atrophy and normal subjects.

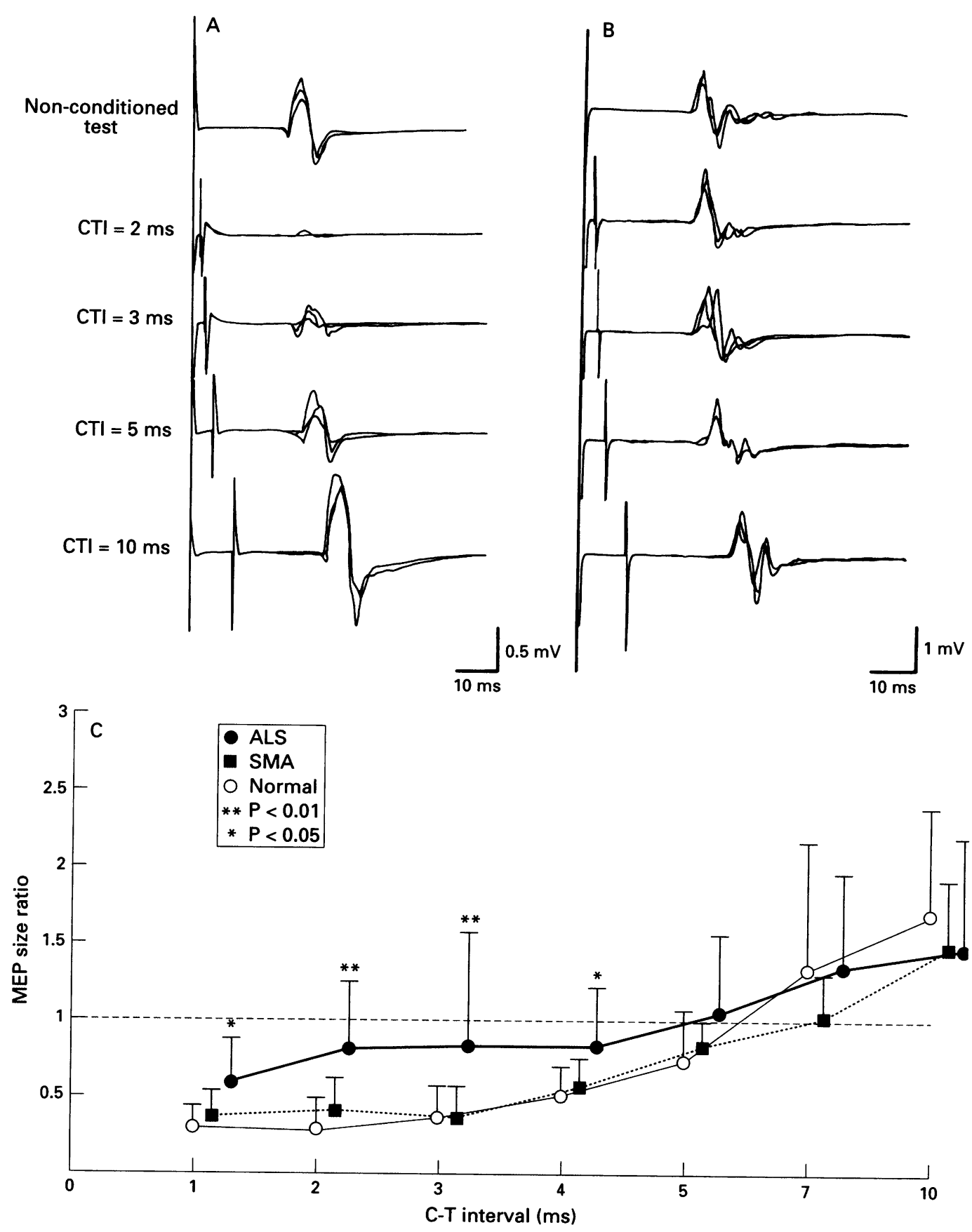

tioned test shocks were suppressed (38.8, $22 \cdot 2,19.9,18 \cdot 8$, and $28.4 \%$ of the response size to non-conditioned test shock respectively). When the test shock was evoked by electrical stimuli, the response to test shocks was not suppressed by the conditioning magnetic stimulus (MEP size ratio 1.06 (SD 0.65) at a C-T interval of $3 \mathrm{~ms}$ ).

For patients with amyotrophic lateral sclerosis, by contrast, the reduction of size of response to test shock by conditioning shock was significant only at the C-T interval of $1 \mathrm{~ms}$, and was much less prominent at 2 and $3 \mathrm{~ms}$ than that in normal subjects $(P<0.01$; fig $1 \mathrm{~B}$ and $1 \mathrm{C})$. The facilitation at $10 \mathrm{~ms}$ was not clear, but the difference from that in the normal subjects was not significant.

For the patients with spinal muscular atrophy the reduction of size of response to test shock by conditioning shock at short C-T intervals was similar to that in the normal conbetween the two groups (fig 1C).

There was no clear correlation between the MEP size ratios at C-T intervals of 2 and $3 \mathrm{~ms}$ and clinical findings, duration of illness, grip strength, and $M$ wave size. STIMULATION ON THE H REFLEX

The effect of subthreshold magnetic cortical stimulation on the $\mathrm{H}$ reflex in the normal controls was facilitation $(P<0.01)$ at $C-T$ intervals of $0-1.5 \mathrm{~ms}$ followed by a rapid decrease in excitation to the baseline. Subsequently, there was a second smaller and longlasting facilitation of the $\mathrm{H}$ reflex. In a patient with amyotrophic lateral sclerosis in whom there was no suppression of the responses on double magnetic cortical stimulation at short C-T trols, and statistically there was no difference

EFFECT OF SUBTHRESHOLD MOTOR CORTICAL 
Figure 2 Effect of subthreshold scalp stimulation on $H$ reflex size. Time courses of the effects as ratios of the mean conditioned to nonconditioned $H$ reflex size in normal controls and one patient with amyotrophic lateral sclerosis.

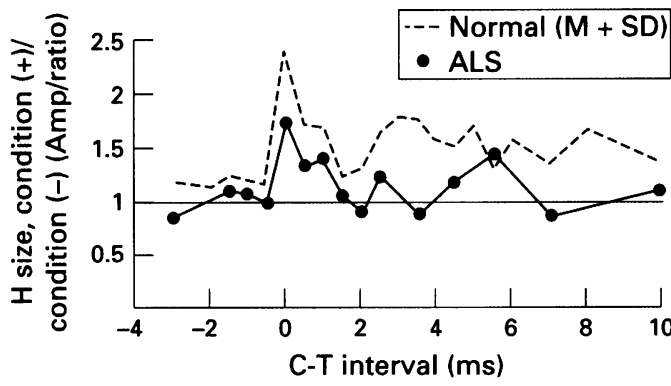

intervals, the effect of the conditioning shock on the $\mathrm{H}$ reflex was in the normal range (fig 2).

\section{Discussion}

Magnetic subthreshold conditioning stimulus over the hand area of the cortex suppresses the responses produced in the relaxed hand muscles by a suprathreshold magnetic test stimulus at short C-T intervals in normal young adults. ${ }^{48}$ Kujirai et $a l^{4}$ suggested that the suppression at C-T intervals of $1-6 \mathrm{~ms}$ is produced by an action on cortical, rather than by spinal, excitability, because subthreshold magnetic conditioning stimuli do not produce a $5 \mathrm{~ms}$ period of inhibition in the forearm $\mathrm{H}$ reflex even though the same stimuli suppress responses evoked in the same muscles by suprathreshold magnetic test stimuli. Moreover, a magnetic conditioning stimulus given over the hand area did not produce suppression of responses evoked in active hand muscles by small anodal electrical stimulation. ${ }^{4}$ Our experiments on normal, aged subjects gave results similar to those of Kujirai et al. ${ }^{4}$ These results indicate that the refractory period of corticospinal axons, after-hyperpolarization in the cell body, or corticospinal recurrent collaterals are not important contributors to this suppression.

In the patients with amyotrophic lateral sclerosis the suppression at short C-T intervals was much less prominent than that in the age matched controls. If the MEP threshold increases in the late stage of amyotrophic lateral sclerosis and the intensity of the conditioning shock increases, newly excited cortical interneurons or fibres may affect suppression even when the mechanism of this suppression is unchanged; but we selected patients with normal MEP thresholds. The reduction of the motor neuron pool seen in our patients with amyotrophic lateral sclerosis probably has little effect on this suppression because it was normal in patients with spinal muscular atrophy who also showed an $M$ wave size that was reduced as much as in amyotrophic lateral sclerosis.

Although the exact site of the activation of the cortical neuron by magnetic stimulation has yet to be determined, the threshold for the I wave is lower than that for the $D$ wave when stimulated with low intensity. ${ }^{111}$ Recent research, however, suggests that even low intensity magnetic stimuli may activate the D wave. ${ }^{12}$ If test stimuli excite the $D$ wave in patients with amyotrophic lateral sclerosis, the suppression should be reduced because of the summation of the $\mathrm{D}$ wave and subliminal increase in the excitability of the $\alpha$-motor neuron by the conditioning ${ }^{413}$; but we selected patients with amyotrophic lateral sclerosis in whom the MEP latency produced by electrical cortical stimulation was shorter by more than $1.0 \mathrm{~ms}$ than that produced by magnetic stimulation when electrical and magnetic stimuli of low intensity were applied during voluntary muscle contraction. This indicates that magnetic test stimuli predominantly activated I waves in our patients with amyotrophic lateral sclerosis. ${ }^{10}$

We do not know the actual site responsible for the lack of the MEP suppression, but speculate that it is at the level of the cerebral cortex, rather than the spinal cord because the effect of the conditioning cortical shock on the $H$ reflex size was in the normal range. Although the impairment of intracortical inhibition in amyotrophic lateral sclerosis seems to be the simplest explanation for the present results, changes in other actions which may contribute to the suppression, such as presynaptic effects on the $\mathrm{H}$ reflex pathway, postsynaptic effects on possible interneurons in the corticospinal pathway, and change of recruitment order of pyramidal tract neurons cannot be excluded. ${ }^{4}$

For some patients with amyotrophic lateral sclerosis, it was not easy to keep their target muscles at rest. Although the electrical silence of the target muscle was confirmed by audio and visual monitorings when double cortical stimuli were applied, we could not monitor the descending discharges of the corticospinal tract which are subliminal for activating $\alpha$-motor neurons. An increase in such descending discharges might contribute to the lack of the MEP suppression because this suppression decreased during voluntary contraction even in normal subjects. ${ }^{7}$ If so, it can be explained as a representation of the hyperexcitability of the pyramidal tract neuron.

There are many hypotheses about the cause of amyotrophic lateral sclerosis. One holds that glutamate, the primary excitatory neurotransmitter in the CNS, accumulates to toxic concentrations at the synapses, causing neurons to die, probably through a calcium dependent pathway. This hypothesis is supported by results of a controlled trial in which the antiglutamate agent reluzole did not stop but slowed down progression of the illness. ${ }^{14}$ In line with this, Rothstein et al ${ }^{15}$ reported decreased high affinity glutamate uptake by synaptosomes from the motor cortex and spinal cord. If true, the increased concentration of extracellular glutamate may cause prolonged overexcitation of pyramidal tract neurons, spinal motor neurons, or both, and this may explain the lack of suppression on double cortical stimulation of our patients with amyotrophic lateral sclerosis. ${ }^{15} \mathrm{We}$ plan to investigate the effect of this antiglutamate agent on the suppression.

1 Eisen A, Seung K, Pant B. Amyotrophic lateral sclerosis (AIS): a phylogenic disease of the corticomotoneuron? Muscle Nerve 1992;15:219-28. 
2 Kaji Y, Kimura J. Editorial comments: amyotrophic latera sclerosis: what makes the direct corticospinal tract so vulnerable? Muscle Nerve 1993;18:872-4.

3 Eisen A. Corticomotoneurons are hyperexcitable early in amyotrophic lateral sclerosis: a magnetic stimulation study. Ann Neurol 1992;32:272.

4 Kujirai T, Caramia MD, Rothwell JC, et al. Corticocortical inhibition in human motor cortex. $\mathcal{f}$ Physiol 1993;471: 501-19.

5 Eisen A, Shytbel W. Clinical experience with transcranial magnetic stimulation. Muscle Nerve 1990;13:995-1011.

6 Cowan JMA, Day BL, Marsden C, Rothwell JC. The effect of percutaneous motor cortex stimulation on $\mathrm{H}$ reflexes of percutaneous motor cortex stimulation on $\mathrm{H}$ reflexes in muscles of the

7 Claus D, Weis $M$, Jahnke U, Plewe A, Brunholzl C. Cortico-spinal conduction studied with magnetic double stimulation in the intact human. $F$ Neurol Sci 1992; 111:180-8.

8 Valls-Sole J, Pascual-Leone A, Wassermann EM, Hallet $M$ Human motor evoked responses to paired transcranial magnetic stimuli. Electroencephalogr Clin Neurophysiol 1992;85:355-64.

9 Linden C, Bruggeman R. Multiple descending corticospinal volleys demonstrated by changes of the wrist flexor H-reflex to magnetic motor cortex stimulation in intact human subjects. Muscle Nerve 1993;16:374-8.

10 Day BL, Dressler D, Noorhout $M$, et al. Electric and mag netic stimulation of human motor cortex: surface EMG and single motor unit responses. F Physiol 1989;412 449-73.

11 Rossini PM, Barker AT, Berardelli A, et al. Non-invasive electrical and magnetic stimulation of the brain, spinal cord and roots: basic principles and procedures for routine clinical application. Report of an IFCN committee. Electroencephalogr Clin Neurophysiol 1994;91:79-92.

12 Burke D, Hicks R, Gandevia SC, Stephen J, Woodforth I, Crawford M. Direct comparison of corticospinal volleys Crawford M. Direct comparison of corticospinal volleys in human subjects to transcranial magn

13 Inghilleri M, Berardelli A, Cruccu G, Priori A, Manfredi M. Motor potential evoked by paired cortical stimuli. M. Motor potential evoked by paired cortical s

14 Bensimon G, Lacomblez L, Meininger V, the ALS Reluzole study group. A controlled trial of Reluzole in amyotrophic lateral sclerosis. $N$ Engl $\mathcal{f}$ Med 1994;330 585-91.

15 Rothstein JD, Mertin LJ, Kunci RW. Deceased glutamate transport by the brain and spinal cord in amyotrophic lateral sclerosis. $N$ Engl f Med 1992;326:1464-8.

\section{HISTORICAL NOTES}

\section{Duane's syndrome}

Students of neurology commonly encounter, but don't always recognise, a type of congenital squint that arises owing to fibrosis in the lateral rectus muscle, which retracts the globe and narrows the palpebral fissure of the eye when it is adducted. The cause of the primary lesion is often unknown. A not uncommon phenomenon, it was reported by Duane.

In 1905 he described the essential features succinctly in the title of his paper ${ }^{1}$ :

"Congenital deficiency of abduction, associated with impairment of adduction, retraction movements, contraction of the palpebral fissure and oblique movements of the eye."

This was later called Duane's retraction syndrome. An autosomal dominant form with four generations described in one kindred was later reported. ${ }^{2}$ There are also associations with deafness and the Klippel Feil anomaly, known as the Wildervanck syndrome, and report of associated congenital defects of the radius.

Alexander Duane (1858-1926) was born in Malone, New York into a distinguished and talented family. A rather authoritarian background probably influenced his work. His father was an army General with a good knowledge of mathematics, which he passed on to his son. His mother, having considerable literary accomplishments, instructed him in English usage that he later employed in contributions to several dictionaries. Duane attended school in Cincinnati and read Medicine at the College of Physicians and Surgeons [Columbia University]. Trained in ophthalmology he practised in New York. In 1903 he translated the Austrian Fuchs's Textbook of Ophthalmology, which ran to eight editions. His work on optics, disordered eye movements, accommodation, and refraction led to election as president of the American Ophthalmological Society in 1924. There were many sides to Duane's character: literary, medical, and naval. He wrote an instructional text on Rules for signalling on land and sea (1899) based on his experience as lieutenant in the Spanish-American war. Later, he served in the first world war as a rather elderly signal officer.

IMS PEARCE 304 Beverley Road, Anlaby, Hull HU10 $7 B G, U K$

1 Duane A Congenital deficiency of abduction, associated with impairment of adduction, retraction movements, with impairment of adduction, retraction movements, ments of the eye. Arch Ophthalmol 1905;34:133-6.

2 Cooper H. A series of cases of congenital ophthalmoplegia externa in the same family. $B M$ f $^{1910 ; 1: 917-8 .}$ 